\title{
Calculation of Failure Probability of Series and Parallel Systems for Imprecise Probability
}

\author{
Bin Suo ${ }^{\mathrm{a}, *}$, Yong-sheng Cheng ${ }^{\mathrm{a},{ }^{*}}$, Chao Zeng ${ }^{\mathrm{a},{ }^{*},} \mathrm{Jun} \mathrm{Li}^{\mathrm{a},{ }^{*}}$ \\ ${ }^{a}$ Institute of Electronic Engineering, China Academy of Engineering Physics, Mianyang, Province, China
}

\begin{abstract}
In the situation that unit failure probability is imprecise when calculation the failure probability of system, classical probability method is not applicable, and the analysis result of interval method is coarse. To calculate the reliability of series and parallel systems in above situation, D-S evidence theory was used to represent the unit failure probability. Multi-sources information was fused, and belief and plausibility function were used to calculate the reliability of series and parallel systems by evidential reasoning. By this mean, lower and upper bounds of probability distribution of system failure probability were obtained. Simulation result shows that the proposed method is preferable to deal with the imprecise probability in reliability calculation, and can get additional information when compare with interval analysis method.
\end{abstract}

Index Terms: reliability; failure probability; series-parallel systems; D-S evidence theory

(C) 2012 Published by MECS Publisher. Selection and/or peer review under responsibility of the Research Association of Modern Education and Computer Science.

\section{Introduction}

In reliability estimation of complex system, it is always in the situation that experimental data is limited, or information is not completed. As sample size is small, and experiment is not enough, there are a variety of uncertainties in the system. In this case, it is hard to calculate the reliability of series-parallel connection units. Traditional methods of uncertainty representation are probability theory and fuzzy theory. Both of them need the probability distributions or membership functions of the unit failure probability. In reality, it is much more easer to get the intervals of unit failure probability than to get their probability distributions. When performance and reliability of system are evaluated in this situation, if the unit failure probability is supposed to follow certain distributions artificially, the results of evaluation are always not objective, and may lead to large analysis errors [1].

Except for probability theory and fuzzy theory, there are a lot of methods to deal with above uncertainty information, such as convex model, info-gap model, interval analysis, and D-S evidence theory.

\footnotetext{
* Corresponding author.

E-mail address: \{suo.y.y, chys_iee, zch_bob \& lijun_iee\} @ 163.com
} 
Convex model is a non-probability method, which represent the uncertainty of parameters with convex model, and translate the uncertainty of input parameters to the system response quality and the problem target quality by performance optimization and robust optimization [2][3]. Info-gap model is developed based on convex model. It is better than other methods in treating with small samples and representing epistemic uncertainty. Info-gap model is consistent with convex model on uncertainty quality representation, but its decision-making approach is better [4-6].

Interval analysis represents the uncertainty by the distribution intervals of parameters, and is very suitable to the situation that the boundaries of parameters are the only valuable information [7-8]. The solutions of interval equations include direct method, combined method, and interval-truncation method. But sometimes their solutions are too pessimistic [9]. In some sense, interval analysis is a part of convex model, because the interval representation of unit failure probability is a special example of convex sets [10].

In D-S evidence theory, belief function is used to represent the precise belief degree of evidence or proposition, and plausibility is used to represent the maximum amount of likelihood that the evidence is true. As D-S evidence theory generalize spot-value function to set function, and its basic research object is set or interval number, it has great advantages in representing and treating with uncertainty information [11-12].

As discussed previous, probability theory and fuzzy theory need the distributions information of unit failure probability, which is hard to obtain in reality. Convex model and info-gap model is available in the situation that the boundaries of parameters are the only useful information, and can draw a relatively valuable conclusion with limited information. But when the boundaries of unit failure probability are known, and multiple information sources about the probability of unit failure probability in there sub-intervals are given as well, these two methods are not available. The information sources may come from different experiments, tests and simulations, or come from the historical data of analogous system, and even the judgments of experts.

To calculate the reliability of series-parallel systems in above situation, D-S evidence theory is used to represent the unit failure probability. Multi-sources information is fused, and belief and plausibility function are used to calculate the reliability of series-parallel systems by evidential reasoning.

\section{D-S Evidence Theory}

Definition 1: $\Theta$ is the whole hypothesis space, which is a non-empty set. If $\mathrm{m}$ is a mass function on $\Theta$, the function $\mathrm{Bel}: 2^{\Theta} \rightarrow[0,1]$ defined by [12]

$$
\operatorname{Bel}(A)=\sum_{B \subseteq A} m(B)
$$

is a belief function, and the function $P l: 2^{\Theta} \rightarrow[0,1]$ defined by

$$
P l(A)=\sum_{B \cap A \neq \varnothing} m(B)
$$

is a plausibility function, where $A \in 2^{\Theta}$ and $A \neq \varnothing$.

They are related to each other by the following equation

$$
\operatorname{Bel}(A)=1-\operatorname{Pl}(\bar{A})
$$

Theorem 1: Let $m_{1}, m_{2}, \cdots, m_{n}$ be the mass functions on $\Theta$, and the focal elements are $A_{i}(i=1,2, \cdots, N)$, then 


$$
m(A)=\left\{\begin{array}{cc}
\frac{1}{1-K} \sum_{\cap A_{i}=A} \prod_{1 \leq i \leq N} m_{i}\left(A_{i}\right) & \mathrm{A} \neq \varnothing \\
0 & \mathrm{~A}=\varnothing
\end{array}\right.
$$

where

$$
K=\sum_{\cap A_{i}=A} \prod_{1 \leq i \leq N} m_{i}\left(A_{i}\right)
$$

Eq. (4) is the Dempster's rule of multi-sources information combination [13].

Theorem 2: Let Bel and Pl be the belief function and plausibility function on $\Theta$ respectively, then

$$
\operatorname{Bel}(A) \leq \operatorname{Pl}(A)
$$

where $A \subset 2^{\Theta}[14]$.

Eq. (5) shows that as a measure of "event $\mathrm{A}$ is true", $\operatorname{Pl}(A)$ is a more optimistic evaluation than $\operatorname{Bel}(A)$. If $P(A)$ is the true value of the measure of set $\{\mathrm{A}$ is true $\}$, then

$$
\operatorname{Bel}(A) \leq P(A) \leq P l(A)
$$

Theorem 3: Consider a set $A \subset \Theta_{X}$, and define a mapping $f: A \rightarrow B$, and $\Theta_{Y}=\left\{B: B=f(A), A \subset \Theta_{X}\right\}$ is another frame of discernment, then

$$
\begin{aligned}
& P l_{Y}(B)=P l_{X}\left(f^{-1}(B)\right) \\
& \operatorname{Bel}_{Y}(B)=\operatorname{Bel}_{X}\left(f^{-1}(B)\right)
\end{aligned}
$$

The proof of (7) and (8) can be seen in Ref. [15].

\section{Calculation of Failure Probability of Series and parallel System}

Suppose each unit in series-parallel system is two-state event, that is, working or failure. Every units are independent each other.

For a system of $n$ units, failure probability of $i^{\text {th }}$ unit is expressed as a function with several basic design parameters

$$
F_{i}=f_{i}\left(x_{i 1}, x_{i 2}, \cdots, x_{i n}\right), \quad i=1,2, \cdots, n
$$

where $x_{i 1}, x_{i 2}, \cdots, x_{i n}$ is the basic design parameters of ith unit. Failure probability of system, $P_{f}$, is

$$
P_{f}=g\left(F_{1}, F_{2}, \cdots, F_{n}\right)
$$

where function $g(\bullet)$ describe the relationship of each units.

For $\mathrm{f} i^{\text {th }}$ unit, because of the uncertainties of design parameters $\mathbf{X}_{\mathbf{i}}=\left\{x_{i 1}, x_{i 2}, \cdots, x_{i n}\right\}, F_{i}$ is imprecise. In practice, the interval value of $F_{i}$ could be obtained by experiments, simulations, and estimates of experts.

Let $\Theta_{F}$ be the frame of discernment of $n$ units $F=\left\{F_{1}, F_{2}, \cdots, F_{n}\right\}$, and frame of discernment of failure probability of system is $\Theta_{P}=\left\{B_{i}: B_{i}=g\left(A_{i}\right), A_{i} \subset \Theta_{F}\right\}$.

For series system, as shown in Fig. 1, failure probability of system is 
$P_{f}=g(\mathbf{F})=1-\prod_{i=1}^{n}\left(1-F_{i}\right)$

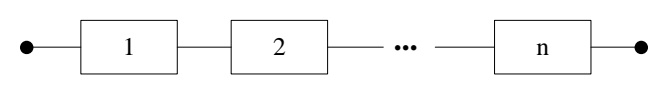

Fig. 1 Series system

For parallel system, as shown in Fig. 2, failure probability of system is

$$
P_{f}=g(\mathbf{F})=\prod_{i=1}^{n} F_{i}
$$

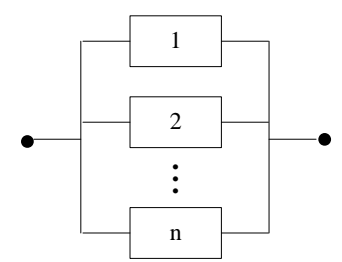

Fig. 2 Parallel system

For parallel-series system, as shown in Fig. 3, failure probability of system is

$$
P_{f}=g(\mathbf{F})=1-\left(1-\prod_{i=1}^{2} F_{i}\right)\left(1-\prod_{i=3}^{4} F_{i}\right)
$$

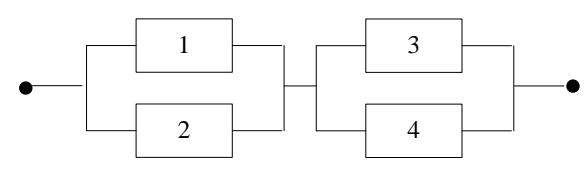

Fig. 3 Parallel-series system

For series-parallel system, as shown in Fig. 4, failure probability of system is

$$
P_{f}=g(\mathbf{F})=\left(1-\prod_{i=1}^{2}\left(1-F_{i}\right)\right)\left(1-\prod_{i=3}^{4}\left(1-F_{i}\right)\right)
$$

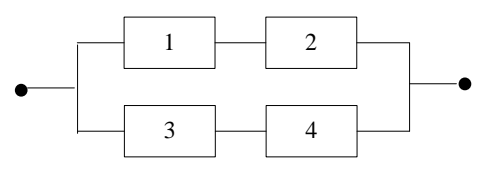

Fig. 4 Series-parallel system

From (7) and (8), then 


$$
\begin{aligned}
& \operatorname{Bel}_{P}\left(p_{f}<p\right)=\operatorname{Bel}_{F}\left(g^{-1}\left(Y_{p}\right)\right)=\sum_{A_{i} \subset g^{-1}\left(Y_{p}\right)} m_{F}\left(A_{i}\right) \\
& \operatorname{Pl}_{P}\left(p_{f}<p\right)=P l_{F}\left(h^{-1}\left(Y_{p}\right)\right)=\sum_{A_{i} \cap f^{-1}\left(Y_{p}\right) \neq \phi} m_{F}\left(A_{i}\right)
\end{aligned}
$$

Where $Y_{p}=\left\{p_{f}: p_{f}<p, p_{f} \in \Theta_{P}\right\}$.

From (15) and (16), the lower and upper bounds of system failure probability can be obtained, where $\operatorname{Bel}_{P}\left(p_{f}<p\right)$ is the lower bound and $P l_{P}\left(p_{f}<p\right)$ is the upper bound.

\section{Numerical Example}

For series-parallel system in Fig. 4, basic belief assignment (BBA) of each unit is shown in table 1. For simplicity, suppose unit 1 is same as unit 2 , and unit 3 same as unit 4.

Table I.BBA of series-parallel system

\begin{tabular}{|c|c|c|c|}
\hline$F_{1}, F_{2}$ & $m\left(x_{1}\right)$ & $F_{3}, F_{4}$ & $m\left(x_{2}\right)$ \\
\hline$[0.003,0.004]$ & 0.5 & {$[0.003,0.005]$} & 0.4 \\
\hline$[0.002,0.005]$ & 0.3 & {$[0.004,0.006]$} & 0.3 \\
\hline$[0.002,0.007]$ & 0.1 & {$[0.002,0.006]$} & 0.2 \\
\hline$[0.001,0.008]$ & 0.1 & {$[0.002,0.007]$} & 0.1 \\
\hline
\end{tabular}

Form (14), system failure probability function is monotone decreasing. So with (15) and (16), lower and upper bounds of failure probability $P_{f}$ is obtained by vertex method [16], as shown in Fig. 5.

From Fig. 2, system failure probability $P_{f}$ is in the interval $\left[7.99 \times 10^{-6}, 2.23 \times 10^{-4}\right]$, and probability of $P_{f}<1.50 \times 10^{-5}$ is 0.03 , probability of $P_{f}<1.73 \times 10^{-4}$ is 0.98 , so $P_{f}$ belong to interval $\left[1.50 \times 10^{-5}, 1.73 \times 10^{-4}\right]$ with confidence of $95 \%$. If interval analysis is used to calculate this issue [8-10], the result is that $P_{f}$ is in the interval $\left[7.99 \times 10^{-6}, 2.23 \times 10^{-4}\right]$, which is similar with D-S evidence method. But there is no additional information can be obtained.

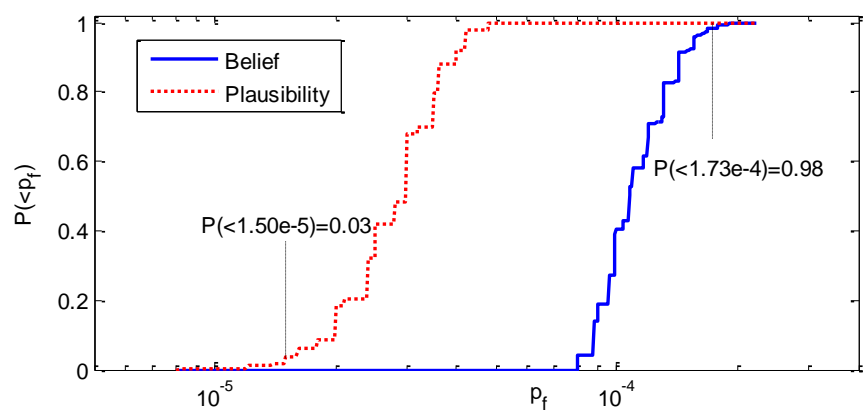

Fig. 5 Probability box of failure probability of Series-parallel system 


\section{Conclusions}

In the situation that unit failure probability is imprecise when calculation the failure probability of system, classical probability method is not applicable, and the analysis result of interval method is coarse. To calculate the reliability of series-parallel systems in above situation, D-S evidence theory was used to represent the unit failure probability. Multi-sources information was fused, and belief and plausibility function were used to calculate the reliability of series-parallel systems by evidential reasoning. By this mean, lower and upper bounds of probability distribution of system failure probability were obtained. The simulation result shows that the proposed method is preferable to deal with the imprecise probability in reliability calculation, and can get additional information when compare with interval analysis method.

In future work, back-up redundancy, k-out-of-n and consecutive k-out-of-n system, which basic units are uncertain, should be studied. Also the failure probability functions of these systems are different from series and parallel systems, but the method to deal with these uncertainties are same by using D-S evidence theory.

\section{References}

[1] Ben-Haim Y, "Convex models of uncertainty in radial pulse buckling of shells," Journal of Applied Mechanics, vol. 60, no. 3, pp. 683-688, March 1993.

[2] Elishakof I, "Essay on uncertainties in elastic and viscoelastic structures: From A.M. Freudenthal's criticisms to modem convex modeling," Computers and Structures, vol. 56, n. 6, pp. 871-895, June 1995.

[3] Ben-Haim Y, “An non-probabilistic concept of reliability," Structural Safety, vol. 14, n. 4, pp. 227-245, April 1994.

[4] Yakov Ben-Haim, "Uncertainty, probability and information-gaps," Reliability Engineering and System Safety, vol. 85, n. 2, pp. 249-266, February 2004.

[5] S. Gareth Pierce, Yakov Ben-Haim, et al. , "Evaluation of Neural Network Robust Reliability Using Information-Gap Theory," IEEE Transactions on Neural Networks, vol. 17, n. 6, pp. 1349-1361, June 2006.

[6] Scott J. Duncan, Bert Bras, Christiaan J.J. Paredis, "An approach to robust decision making under severe uncertainty in life cycle design," International Journal of Sustainable Design, vol. 1, n. 1, pp. 45-59, January 2008.

[7] Qiu Zhiping, Chen Suhuan, Elishakof I, "Non-probabilistic eigenvalue problem for structures with uncertain parameters via interval analysis," Chaos, Solitons and Fractals, vol. 7, n. 3, pp. 303-308, March 1996.

[8] Weidong Wu, S.S. Rao, "Uncertainty analysis and allocation of joint tolerances in robot manipulators based on interval analysis," Reliability Engineering and System Safety, vol. 92, n. 1, pp. 54-64, January 2007.

[9] C. Jiang X. Han, F. J. Guan, Y. H. Li, "An uncertain structural optimization method based on nonlinear interval number programming and interval analysis method," Engineering Structures, vol. 29, n. 11, November 2007.

[10] Fahed Abdallah, Amadou Gning, Philippe Bonnifait, "Box particle filtering for nonlinear state estimation using interval analysis," Automatica, vol. 44, n. 3, March 2008.

[11] D. Harmance, G. J. Klir, "Measuring total uncertainty in Dempster-Shafer theory: A novel approach," International Journal of General Systems, vol. 22, n. 4, pp. 405-419, April 1997.

[12] R. R. Yager, “Arithmetic and other operations on Dempster-Shafer structures," International Journal of Man-machine Studies, vol. 25, pp. 357-366, June 1986.

[13] Dempster A P, "Upper and lower probabilities induced by a multi valued mapping," Annals Math Statist, vol. 38, no. 2, pp. 325-339, 1967.

[14] Shafer G. A, Mathematical Theory of Evidence. Princeton University Press, 1976.

[15] C. Joslyn, J.C. Helton, "Bounds on belief and plausibility of functionality propagated random sets," 2002 Annual Meetings of the North American Fuzzy Information Processing Society, pp.27-29, June 2002, New 
Orleans, LA, IEEE, Piscataway, NJ, pp:412-417, 2002.

[16] Harish Agarwal, John E. Renaud, Evan L, "Preston, et al. Uncertainty quantification using evidence theory in multidisciplinary design optimization," Reliability Engineering and System Safety, vol. 85, n. 1, pp. 281-294, January 2004. 